\title{
Identification Procedures as Tools for Fault Diagnosis of Rotating Machinery
}

\author{
DIPL.-ING. SUSANNE SEIBOLD \\ Lehrstuhl für Technische Mechanik, Universität Kaiserslautern, D-67653 Kaiserslautern, Germany \\ PROF. DR.-ING. CLAUS-PETER FRITZEN \\ Institut für Mechanik und Regelungstechnik, Universität-GH Siegen, D-57076 Siegen, Germany
}

\begin{abstract}
System identification procedures offer the possibility to correct erroneous models, based on measurement data. Recently, this conventional field of application is being extended to fault detection and system diagnosis. In contrast to conventional approaches, identification procedures try to establish an unequivocal relation in between the damage and specific mechanical parameters, based on a suitable model. Furthermore, they can be employed during normal operation of the machinery. In this paper, several identification procedures on the basis of the Extended Kalman Filter are introduced and employed for model-based fault detection. Their feasability is proved by several examples. First, it is shown that the crack depth of a simulated Jeffcott-rotor can be calculated correctly. Then, the procedures are utilized to determine the crack depth of a rotor test rig. Finally, it is proved that identification procedures can be employed for the determination of unbalances without having to apply test masses.
\end{abstract}

Key Words Fault diagnosis; System identification; Kalman filtering; Damage supervising; Combined state and parameter estimation; Balancing

\section{INTRODUCTION}

M onitoring and diagnosis of machinery is a field which is receiving increasing interest. Current practices and trends are described e.g. by Eshleman [1990], Randall [1990], Jonas [1992] and El-Shafei [1993]. Very often, special attention is being focused on the detection of cracks: Wauer [1990] gives a concise overview about the research performed in the area of modeling the dynamics of cracked rotors and detection procedures. A possibility to detect a crack is e.g. the monitoring of the rotor vibrations and the comparison to the results of a suitable analytical model, Muszynska [1982], or to a reference signal, Imam [1983].

In contrast to approaches utilizing signal analysis, model-based procedures are being developed, which try to establish an unequivocal relation in between the damage (not necessarily a crack) and specific mechanical parameters. To achieve this, a-priori knowledge like e.g. a mathematical description of the system dynamics is employed. Waller and Schmidt [1990] describe a modal observer suitable for the application to MDOF systems. Another method is presented by Söffker and Bajkowski [1991], which makes use of the state observer for nonlinear systems developed by Müller [1990]. Structural similarities to the approach of Fritzen and Seibold [1990] can be shown, where the Extended Kalman Filter is applied to shaft crack detection.

In this paper, time domain identification algorithms, based on the Extended Kalman Filter (EKF) and the Instrumental Variables method (IV), and a modification of the EKF in analogy to Müller [1990] are presented and applied to fault diagnosis of rotating machinery. Specific advantages are the direct processing of time domain measurements, without the necessity of a transformation to frequency domain, and the establishing of a physically meaningful relation in between the damage and a certain parameter. Furthermore, unknown states are recon- 
structed and possible parameter changes can be monitored during normal operation of the machinery. These model-based procedures are sensible supplementals of the approved tools for fault diagnosis and their feasability is explained by two examples: a simulated Jeffcottrotor, where the crack depth is identified, and a rotor test rig, where the depth of a crack and the unbalance are determined.

\section{THE INSTRUMENTAL VARIABLES METHOD (IV)}

The IV-method is a parameter identification procedure which yields consistent and asymptotically unbiased estimates, requiring only little information about the noise characteristics. For a general description see Durbin [1954]. Wong and Polak [1967] and Young [1970] apply the IV-method as a purely statistical procedure to control problems. The IV-method can be derived from the well known Least Squares normal equations

$$
\mathrm{A}^{\mathrm{T}} b-\mathrm{A}^{\mathrm{T}} \mathrm{A} \hat{p}_{\mathrm{LS}}=0
$$

A being the coefficient matrix. Eq. (1) can be established for problems that are linear in the parameters. The estimated parameters $\hat{p}_{\mathrm{LS}}$, which result from (1), are biased, and the expected values of $\hat{p}_{\mathrm{LS}}$ are $\mathrm{e}$

$$
\mathrm{E}\left\{\hat{p}_{\mathrm{LS}}\right\}=p_{0}+\mathrm{E}\left\{\left(\mathrm{A}^{\mathrm{T}} \mathrm{A}\right)^{-1} \mathrm{~A}^{\mathrm{T}} \epsilon\right\}
$$

$p_{0}$ are the true parameters and $\epsilon$ is the error vector. The bias $\mathrm{E}\left\{\left(\mathrm{A}^{\mathrm{T}} \mathrm{A}\right)^{-1} \mathrm{~A}^{\mathrm{T}} \epsilon\right\}$ will not vanish, because $\mathrm{A}$ and $\epsilon$ are usually correlated. Nevertheless, unbiased estimates can be produced if an Instrumental Variable matrix W with certain properties is introduced. According to Young [1970], the elements of W should be highly correlated with the unobservable noise-free responses of the system, but totally uncorrelated with the noise, so that $\mathrm{E}\left\{\mathrm{W}^{\mathrm{T}} \epsilon\right\}=0$ and $\mathrm{E}\left\{\mathrm{W}^{\mathrm{T}} \mathrm{A}\right\}$ is existing and nonsingular. Then, unbiased estimates $\hat{p}_{\mathrm{IV}}$ are be obtained by

$$
\hat{p}_{\mathrm{IV}}=\left(\mathrm{W}^{\mathrm{T}} \mathrm{A}\right)^{-1} \mathrm{~W}^{\mathrm{T}} b
$$

Wong and Polak [1967] and Young [1970] give a recursive version of the IV-method (RIV):

$$
\begin{aligned}
& \hat{p}_{\mathrm{k}+1}=\hat{p}_{\mathrm{k}}+\gamma_{\mathrm{k}}\left[y_{\mathrm{k}+1}-\mathrm{A}_{\mathrm{k}+1}^{\mathrm{T}} \hat{p}_{\mathrm{k}}\right], \\
& \gamma_{k}=\left[\mathrm{A}_{k+1}^{\mathrm{T}} \mathrm{P}_{k} \mathrm{~W}_{k+1}+\mathrm{I}\right]^{-1} \mathrm{P}_{k} \mathrm{~W}_{k+1},
\end{aligned}
$$

$$
P_{k+1}=\left[I-\gamma_{k} A_{k+1}^{T}\right] P_{k} .
$$

In the case of a mechanical system, assuming that displacements $x$ are measured, we need the complete information about $x$ and its derivatives $\dot{\mathrm{x}}$ and $\ddot{\mathrm{x}}$ and to determine the coefficient matrix $\mathrm{A}$ and the Instrumental Variables matrix W. These derivatives could be calculated by means of numerical differentiation. But, this might lead to bad results, especially if the level of measurement noise is high. Another possibility is to apply spline approximation, which is a better choice. Of course, this might cause problems if stochastic signals are dealt with. In this paper, it will be shown how the Extended Kalman Filter can be employed, cf. Seibold $e t$ al. [1993].

\section{THE EXTENDED KALMAN FILTER (EKF)}

The EKF is based on the Kalman Filter (KF), which was derivated by Kalman [1960] based on the concept of orthogonal projection, as an optimal filter for linear systems. But, as for many nonlinear problems cannot be linearized globally, suitable algorithms have to be developed. Jazwinski [1970] describes, how the KF has to be modified so that it can be applied as EKF to nonlinear systems. The basic idea is to linearize at each time step around a reference trajectory. In this way, the global nonlinearity is maintained.

The motion of a mechanical system can be described by the differential equation

$$
\mathrm{M}(x, p, \mathrm{t}) \ddot{x}=g(x, \dot{x}, u, p, \mathrm{t}),
$$

$\mathrm{M}$ being the mass matrix which might depend on the displacements $x$ and time t. $g$ is the vector of generalized forces and moments, including elastic, damping, gyroscopic forces etc., and $u$ is the input vector.

The EKF is a recursive time domain procedure suitable for the identification of nonlinear systems. System disturbances as well as measurement noise can be considered and a state space formulation of the differential equations of motion is required. In the case of mechanical systems, the state space vector $z$ consists of the displacements $x$ and the velocities $\dot{x}$. For the purpose of simultaneous state and parameter estimation, $z$ is extended by the unknown parameters $p$ :

$$
z^{\mathrm{T}}=\left(x^{\mathrm{T}}, \dot{x}^{\mathrm{T}}, p^{\mathrm{T}}\right)=\left(z_{\mathrm{x}}^{\mathrm{T}}, z_{\mathrm{V}}^{\mathrm{T}}, z_{\mathrm{p}}^{\mathrm{T}}\right) .
$$

Applying state space notation, the vibrations of a mechanical system can be described by the following equation, assuming time constant parameters: 
$\dot{z}=\left[\begin{array}{c}x \\ \dot{x} \\ p\end{array}\right]=\left[\begin{array}{c}f_{\mathrm{x}} \\ f_{\mathrm{v}} \\ f_{\mathrm{p}}\end{array}\right]=\left[\begin{array}{c}z_{v} \\ \mathrm{M}^{-1}\left(z_{\mathrm{x}}, \mathrm{t}\right) g\left(z_{\mathrm{x}}, z_{\mathrm{v}}, z_{\mathrm{p}}, u, \mathrm{t}\right) \\ 0\end{array}\right]$.

Considering discrete time steps $t_{k}$, the relation in between the observed measurement data $y_{\mathrm{k}}$ and the state variables $z_{\mathrm{k}}$ at time $\mathrm{t}_{\mathrm{k}}$ is

$$
y_{\mathrm{k}}=\mathrm{C} z_{\mathrm{k}}+n_{\mathrm{k}}
$$

In our case, $\mathrm{C}$ is a constant matrix and $n_{\mathrm{k}}$ denotes the measurement noise. The EKF consists of a predictor and a corrector part, with the prediction

$$
\hat{z}_{\mathrm{k}+1 / \mathrm{k}}=\hat{z}_{\mathrm{k}}+\int_{\mathrm{t}_{\mathrm{k}}}^{\mathrm{t}_{\mathrm{k}+1}} f\left(\hat{z}_{\mathrm{k}}, \mathrm{t}_{\mathrm{k}}, u_{\mathrm{k}}\right) \mathrm{dt}
$$

basing on the model equations $f$. The corresponding covariance matrix is

$$
\mathrm{P}_{k+1 / k}=\mathrm{A}_{k}^{*} \mathrm{P}_{k} \mathrm{~A}_{k}^{*^{\mathrm{T}}}+\mathrm{Q}_{k}^{*}
$$

Employing new measurements $y_{\mathrm{k}+1}$, the prediction (11) is corrected by

$$
\hat{z}_{\mathrm{k}+1}=\hat{z}_{\mathrm{k}+1 / \mathrm{k}}+\mathrm{K}_{\mathrm{gk}+1}\left(y_{\mathrm{k}+1}-\mathrm{C} \hat{z}_{\mathrm{k}+1 / \mathrm{k}}\right)
$$

and the corrected covariance matrix is

$\mathrm{P}_{\mathrm{k}+1}=\left(\mathrm{I}-\mathrm{K}_{\mathrm{gk}+1} \mathrm{C}\right) \mathrm{P}_{\mathrm{k}+1 / \mathrm{k}}\left(\mathrm{I}-\mathrm{K}_{\mathrm{gk}+1} \mathrm{C}\right)^{\mathrm{T}}+\mathrm{K}_{\mathrm{gk}+1} \mathrm{RK}_{\mathrm{gk}+1}^{\mathrm{T}}$.

The Kalman gain matrix $\mathrm{K}_{\mathrm{g}}$ is calculated by

$$
\mathrm{K}_{\mathrm{gk}+1}=\mathrm{P}_{\mathrm{k}+1 / \mathrm{k}} \mathrm{C}^{\mathrm{T}}\left(\mathrm{CP}_{\mathrm{k}+1 / \mathrm{k}} \mathrm{C}^{\mathrm{T}}+\mathrm{R}_{\mathrm{k}}\right)^{-1}
$$

The initial values are normally distributed, with mean $z_{0}$ and covariance $P_{0} . A_{k}^{*}$ is the time discrete system matrix and results from a local linearization of the system equations $f$ around the estimated trajectory at each time step $t_{k}$ :

$$
\begin{aligned}
& A_{k}^{*}=\exp ^{A_{h} \Delta t}=I+A_{k} \Delta t+A_{k}^{2} \Delta t^{2} / 2 !+\ldots ; \Delta t=t_{k+1}-t_{k}, \\
& \mathrm{~A}_{\mathrm{k}}=\frac{\partial f}{\partial z^{\mathrm{T}}} \mid z=\hat{z}_{\mathrm{k}}
\end{aligned}
$$

It is important to note that in this way, the global nonlinearity is maintained. $\mathrm{Q}_{\mathrm{k}}$ is the discrete system noise covariance matrix and has to be calculated at each time step $t_{k}$, too. $R_{k}$ is the covariance matrix of the measurement noise $n_{\mathrm{k}}$. Therefore, the confidence in the initial values $z_{0}$, in the system equations $f$ as well as in the measurements $y_{\mathrm{k}}$ can be expressed by means of the covariance matrices $\mathrm{P}, \mathrm{Q}$ and $\mathrm{R}$, respectively.

\section{THE COMBINED ALGORITHM REKFIV}

The EKF is very time consuming and might, in certain cases, show poor convergence properties if it is used for combined state and parameter estimation, Ljung [1979]. But, being used exclusively as a state estimator, the EKF will perform well and provide us with the advantage of the reconstruction of unknown states and dofs. Therefore, the idea is to combine EKF and RIV in a way that the EKF is used as a state estimator to produce the Instrumental Variables for the RIV. We propose to proceed in the following manner, see figure 1:

1. Estimate the initial values by recursive Least Squares (RLS). In the case of measured displacements $x$, calculate the lacking velocities $\dot{x}$ and accelerations $\ddot{x}$ by spline approximation. This leads to a complete matrix A.

2. Use the EKF as a state estimator only, i.e. omit line three in eq. (9). The parameters are set to the initial values calculated above and remain constant.

3. The filtered time series produced by the state-EKF are used to build up the IV-matrix W. The lacking accelerations can be derived via the system equations $f$, see eq. (9). In the case of system noise respectively

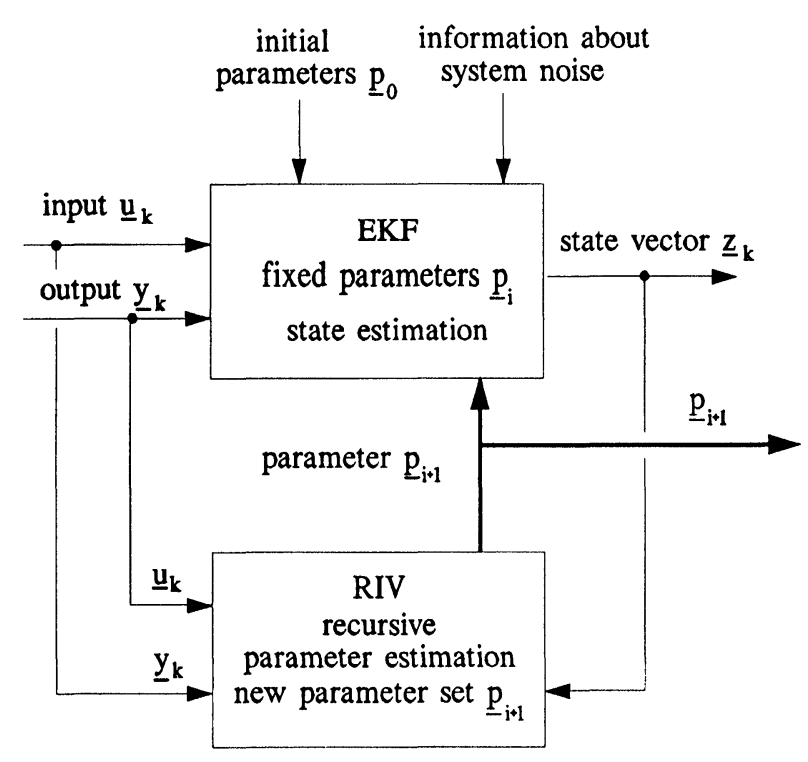

FIGURE 1 Recursive combination of EKF and RIV: REKFIV. 
erroneous models, the covariance matrix $Q$ is calculated.

4. Now, with complete matrices A and W, an improved set of parameters $p$ is determined.

5. Repeat from 2. on as long as measurement data is available or until the parameters converge.

\section{THE MODIFIED EXTENDED KALMAN FILTER (MEKF)}

The EKF, (11)-(17), was developed for stochastic dynamic systems and shows a structural similarity to the state observer, which is known from control theory. Müller [1990] designed a state observer for deterministic systems in order to determine unknown nonlinearities. These are interpreted as "external disturbances", which are characterized by a suitable model. In analogy, the EKF is modified in such a manner that it can reconstruct these "external disturbances", Seibold et al. [1993], without the necessity to model their dynamics, Söffker et al. [1994]. Essentially, the state space vector $z$ has to be extended by the "external disturbances" and their time derivatives $\left(v^{\mathrm{T}}, \dot{v}^{\mathrm{T}}\right)$ :

$$
z^{\mathrm{T}}=\left(x^{\mathrm{T}}, \dot{x}^{\mathrm{T}}, v^{\mathrm{T}}, \dot{v}^{\mathrm{T}}\right)
$$

The EKF-equations can be used like being described in (11)-(17). The coupling in between the linear part of the system $\left(x^{\mathrm{T}}, \dot{x}^{\mathrm{T}}\right)$ and the "exogeneous system" $\left(v^{\mathrm{T}}, \dot{v}^{\mathrm{T}}\right)$ is done via the prediction (11), where the linear part of the system has to be supplemented by the exogeneous system, which was calculated in the preceding time step.

\section{EXAMPLES}

\section{Simulated Example: Jeffcott Rotor with a Transversal Crack}

In our first example, the identification procedures will be used to detect a transverse crack in a Jeffcott-rotor. The crack is considered by the breathing crack model of Gasch [1976]. The system rotates with circular frequency $\omega$, is viscously damped and loaded by unbalance and gravitational forces, figure 2 . As a consequence of the opening and closing due to the crack, the stiffness $k_{\xi}$ switches in between the two values $\mathrm{k}_{\xi 1}$ and $\mathrm{k}_{\xi 2}$, depending on the actual displacements $x_{1}$ and $x_{2}$ (resp. $\xi$ ), figure 3. The stiffness of the uncracked shaft is $k_{0}$ and for small cracks, we can assume $\mathrm{k}_{\xi 2}=\mathrm{k}_{0}=\mathrm{k}_{\eta}$. In rotating fixed $x_{1}-x_{2}-$ coordinate system rotating $\xi-\eta$-coordinate system
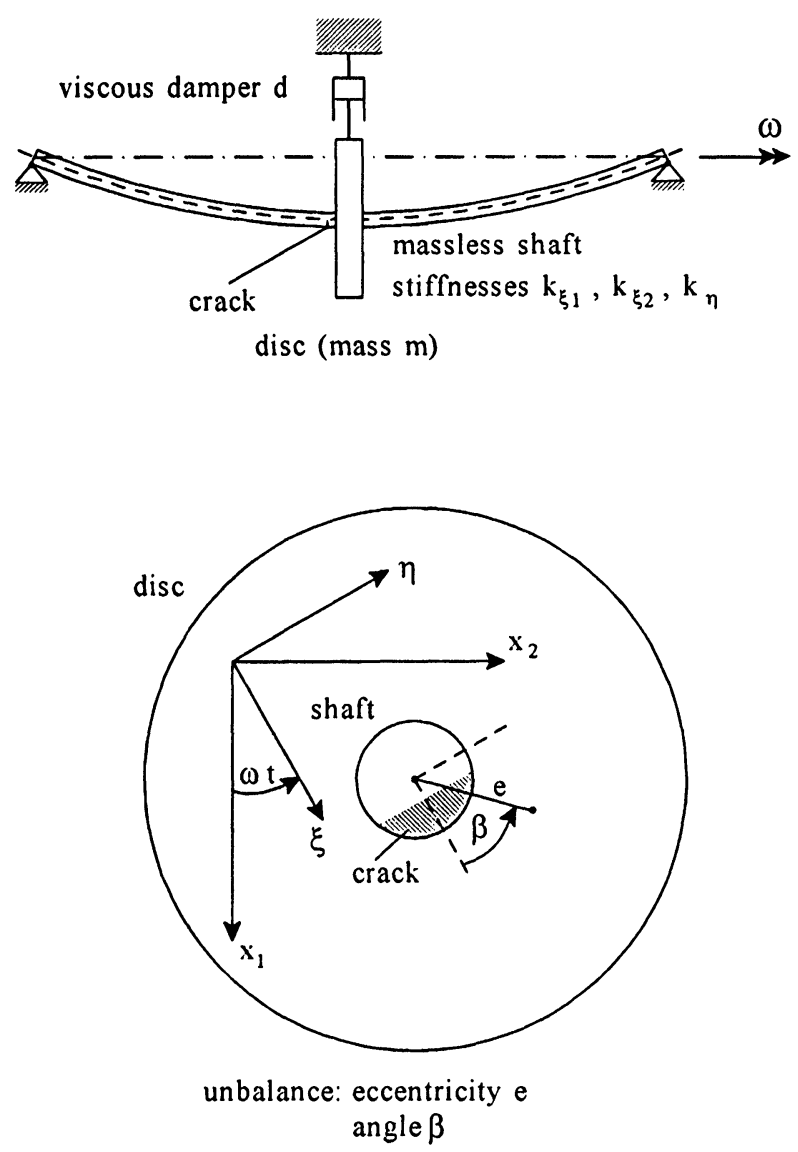

FIGURE 2 Jeffcott-rotor with transversal crack.

$\xi$ - $\eta$-coordinates, introducing well-suited reference quantities (static displacement $\mathrm{x}_{1 \mathrm{~s}}$ of the uncracked rotor, eigenfrequency $\omega_{0}$ of the uncracked shaft), the system equations are the following:

$$
\begin{aligned}
& {\left[\begin{array}{l}
\xi \\
\eta
\end{array}\right]^{\prime \prime}+2\left[\begin{array}{cc}
\mathrm{D} & -v \\
v & \mathrm{D}
\end{array}\right]\left[\begin{array}{l}
\xi \\
\eta
\end{array}\right]^{\prime}+\left[\begin{array}{cc}
\nu_{\xi \mathrm{i}}^{2}-v^{2} & -2 \mathrm{D} v \\
2 \mathrm{D} v & v_{\eta}^{2}-v^{2}
\end{array}\right]\left[\begin{array}{c}
\xi \\
\eta
\end{array}\right]} \\
& =\left[\begin{array}{c}
\cos v t \\
-\sin v t
\end{array}\right]+\in v^{2}\left[\begin{array}{c}
\cos \beta \\
\sin \beta
\end{array}\right],
\end{aligned}
$$

where $\mathrm{D}$ is the dimensionless damping factor, $\tau=\omega_{0} \mathrm{t}$ is the dimensionless time, $v_{\xi \mathrm{i}}^{2}=\mathrm{k}_{\xi \mathrm{i}} / \mathrm{k}_{0}(\mathrm{i}=1$ : opened crack for $\xi>0 ; i=2$ : closed crack for $\xi<0$ ) and $\nu_{\eta}^{2}=$ $\mathrm{k}_{\eta} / \mathrm{k}_{0}$ are the squares of the related eigenfrequencies, and the dimensionless rotational speed is $v=\omega / \omega_{0}$. The eccentricity $\epsilon=\mathrm{e} / \mathrm{x}_{1 \mathrm{~s}}$ as well as the coordinates $\xi$ and $\eta$ are related to the static displacement $x_{1 S}$. (..)' is the 

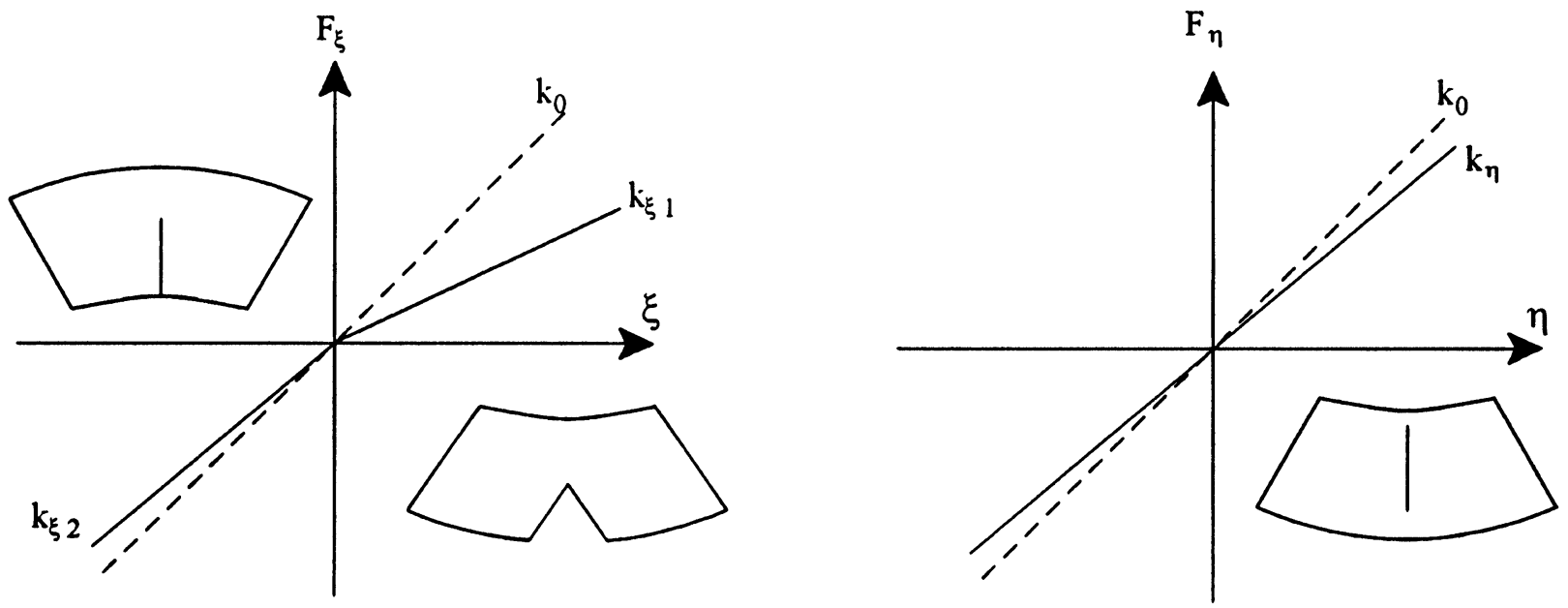

FIGURE 3 Relation in between force and displacement.

derivative with respect to $\tau$. The switching of the suffness $k_{\xi}(\xi)$ can be expressed by

$$
k_{\xi}=k_{\xi 2}+\left(k_{\xi 1}-k_{\xi 2}\right)\left(\frac{1}{2}+\frac{1}{\pi} \arctan \left(\mathrm{C}_{0} \xi\right)\right) ; \mathrm{C}_{0}>0
$$

cf. Fritzen, Seibold [1990], and the decreasing of the stiffness due to the crack is described by the crack factor r:

$$
\mathrm{r}=\frac{\mathrm{k}_{0}}{\mathrm{k}_{\xi 1}}-1=\left(\frac{1}{v_{\xi 1}}\right)^{2}-1
$$

Transforming (19) to fixed $\mathrm{x}_{1}-\mathrm{x}_{2}$-coordinates yields

$$
\begin{aligned}
& {\left[\begin{array}{c}
x_{1} / x_{1 S} \\
x_{2} / x_{1 S}
\end{array}\right]^{\prime \prime}+2 D\left[\begin{array}{l}
x_{1} / x_{1 S} \\
x_{2} / x_{1 S}
\end{array}\right]^{\prime}+\left[\begin{array}{l}
x_{1} / x_{1 S} \\
x_{2} / x_{1 S}
\end{array}\right]+} \\
& {\left[\begin{array}{cc}
-\cos ^{2} v \tau & -\sin v \tau \cos v \tau \\
-\sin v \tau \cos v \tau & -\sin ^{2} v \tau
\end{array}\right] \Delta\left[\begin{array}{l}
\mathrm{x}_{1} / \mathrm{x}_{1 \mathrm{~S}} \\
\mathrm{x}_{2} / \mathrm{x}_{1 \mathrm{~S}}
\end{array}\right]=} \\
& v(\tau) \\
& =\left[\begin{array}{l}
1 \\
0
\end{array}\right]+\frac{\epsilon}{x_{1 S}} v^{2}\left[\begin{array}{l}
\cos v \tau \cos \beta-\sin v \tau \sin \beta \\
\sin v \tau \cos \beta-\cos v \tau \sin \beta
\end{array}\right] \text {, }
\end{aligned}
$$

$f(\tau)$

using the relation

$$
\Delta=\frac{\mathrm{r}}{\mathrm{r}+1}\left(\frac{1}{2}+\frac{1}{\pi} \arctan \left(\mathrm{C}_{0} \xi\right)\right)
$$

The equations of motion (22) can be formulated in a different way, denoting

$$
\begin{gathered}
x^{\mathrm{T}}=\left[\mathrm{x}_{1} / \mathrm{x}_{1 \mathrm{~S}} \mathrm{x}_{2} / \mathrm{x}_{1 \mathrm{~S}}\right]: \\
x^{\prime \prime}+2 \mathrm{D} x^{\prime}+x=f(\tau)-v(\tau) .
\end{gathered}
$$

The linear part of the equations of motion is on the left hand side of (24), and on the right hand side are the excitations $f(\tau)$ and the nonlinear part $v(\tau)$, which can be interpreted as additional forces due to the crack. The dynamic behaviour is simulated by numerical integration of (22). The parameters are set to $\mathrm{D}=0.011, v=0.45$, $\tau=0.01, \epsilon=0.01$ and $\beta=0$. The relation of the crack factor $\mathrm{r}$ to the crack depth can be derived e.g. according to Mayes and Davies [1984]: $r=0.01$ corresponds to a crack depth in percentage of the diameter of the shaft of $19 \%$. On the basis of measured displacements $x_{1}$ and $x_{2}$, EKF as state and parameter estimator, REKFIV and MEKF are employed to detect the depth of the crack.

For the application of EKF and REKFIV, the knowledge of the crack model $v(\tau)$, eq. (22), is assumed. EKF and REKFIV calculate a crack factor $r=0.00995$ resp. $r=0.01005$, figure 4 . The initial parameters were set to $\mathrm{r}_{0}=0$, which implies that no crack is present. In contrast to this, the calculations with the MEKF can be performed without the knowledge of a crack model, i.e. the exogeneous system $v(\tau)$ does not have to be structured. The result of the MEKF is shown in figure 5, where it is compared to the true values $v_{1}(\tau)$ according to eq. (22). This exogeneous system can be interpreted as nonlinear forces due to the crack. Except for small deviations at the first time steps, the two curves are identical. The quantitative result of $r=0.01$, of course, can only be derived by a model of the crack, in this case 


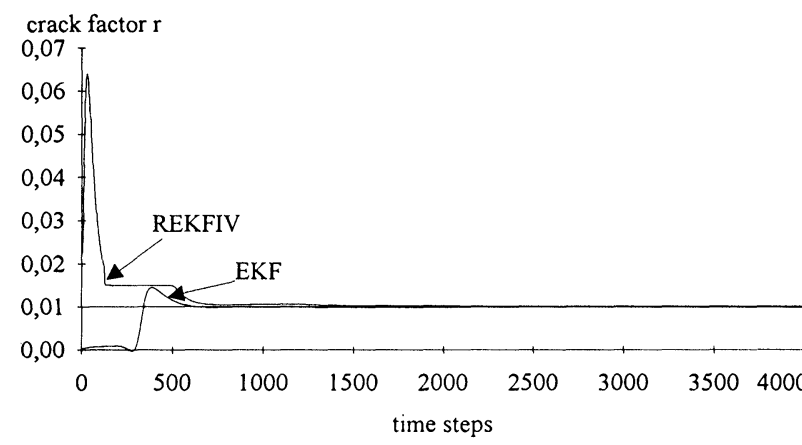

FIGURE 4 Identification of the crack factor $r$ by EKF and by REKFIV in comparison to the true value $r=0.01$; simulated example.

according to eq. (22). Furthermore, this is supplemented by a qualitative description of the nonlinearity: the opening $\left(\mathrm{v}_{1}(\tau)>0\right)$ and closing $\left(\mathrm{v}_{1}(\tau)=0\right)$ of the crack can be seen in figure 5 , too.

The EKF can be modified to estimate time variable parameters, i.e. in this case time dependent stiffnesses, so that a crack model is not a prerequisite for the calculations. In the EKF-equation (12), the covariance matrix of system noise $\mathrm{Q}$ has to be set to adequate values. Figure 6 shows the identification of one of the time varying stiffness parameters. Like in figure 5 , the opening $\left(\mathrm{c}_{11}<\right.$ 1) and closing $\left(c_{11}=1\right)$ of the crack can be seen. The results correspond to the true crack factor $r=0.01$ and are compared to the true slope of the stiffness $\mathrm{c}_{11}=1$ $-\cos ^{2} \nu \tau \Delta$, see eq. (22). The two curves plottet in figure 6 are almost identical.

\section{Rotor Test Rig: Identification of the Crack Depth}

The rotor test rig, figure 7 , consists of a long, thin shaft of $18 \mathrm{~mm}$ diameter on hinged supports. A disc is mounted in the middle. Initiated by a slot with a depth of $2 \mathrm{~mm}$, a crack of $1 \mathrm{~mm}$ is introduced, so that the total depth of the damage is $3 \mathrm{~mm}$ respectively $17 \%$ of the

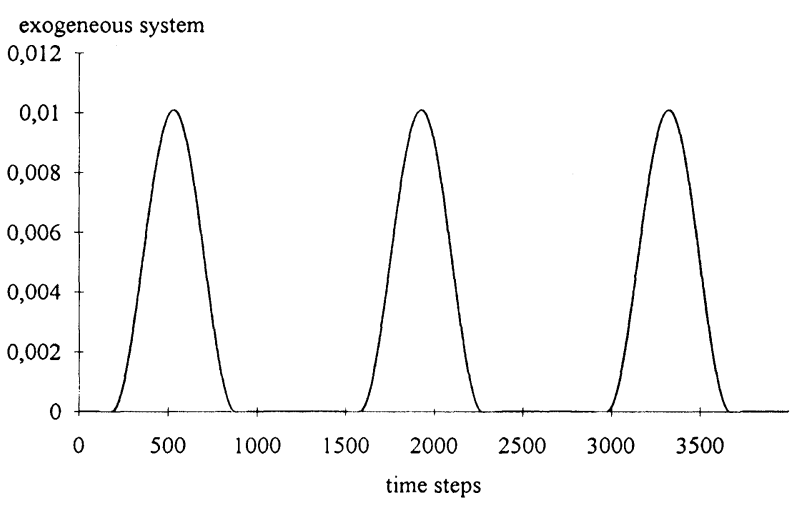

FIGURE 5 Identification of the nonlinear forces (exogeneous system) due to the crack by MEKF, in comparison to the true slope of $v_{1}(\tau)$; simulated example.

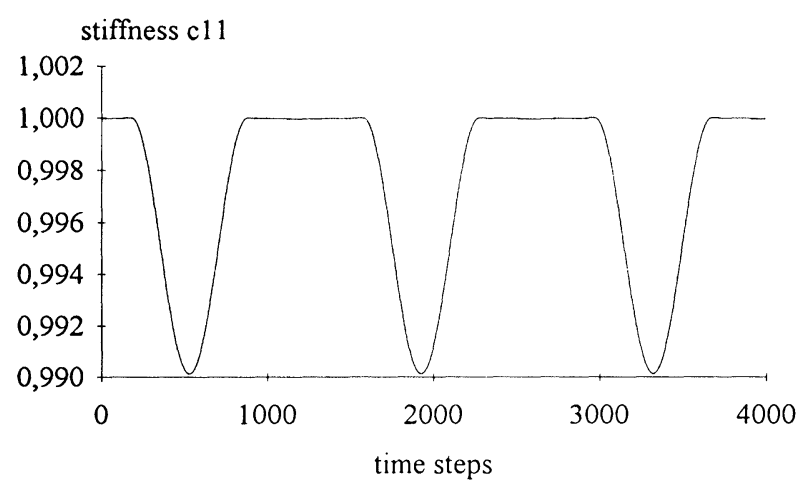

FIGURE 6 Identification of the time varying stiffness $c_{11}(\tau)$ in comparison to the true slope; simulated example.

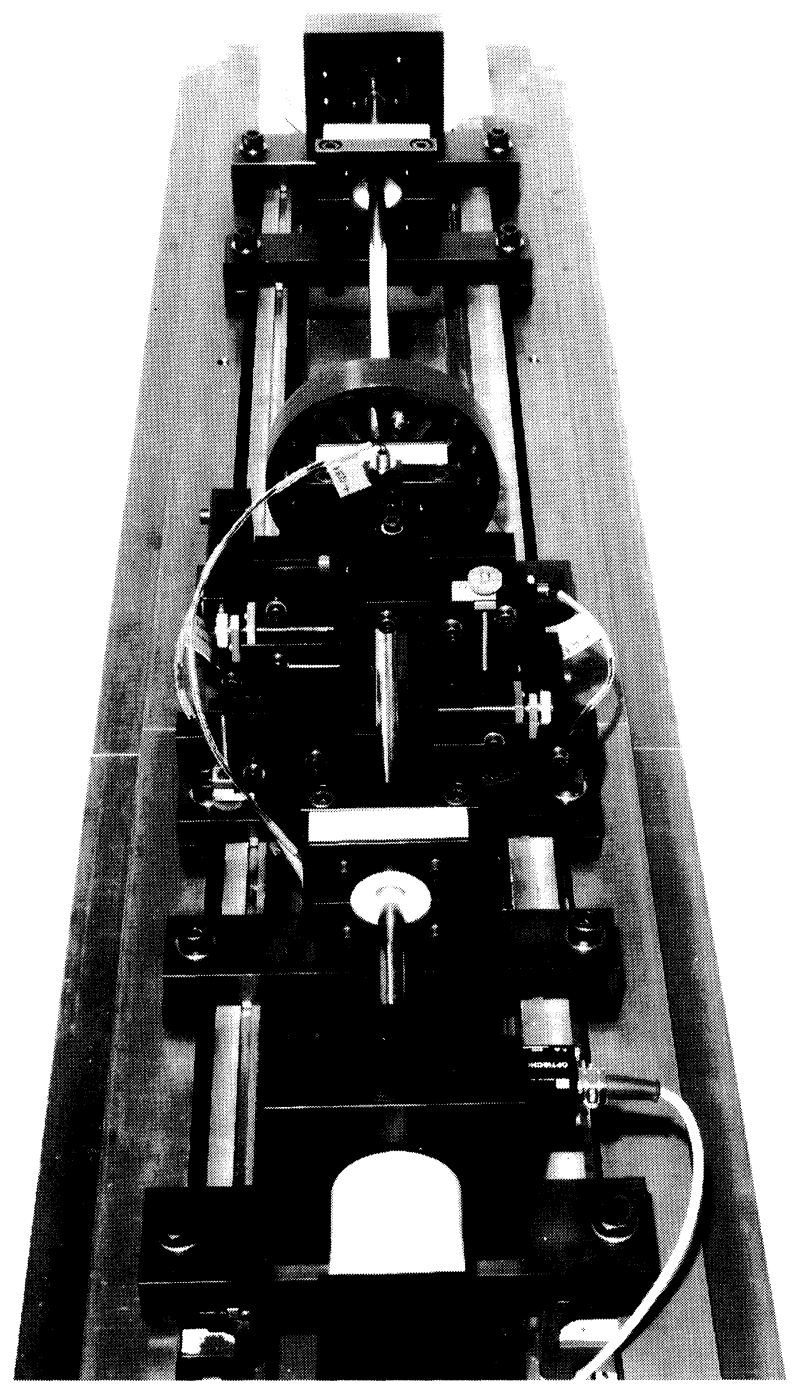

FIGURE 7 Rotor test rig 
diameter of the shaft. Figure 8 shows the fractured cross section. A beach mark, which results in a dark line on the crack face, was set by applying a static overload in order to be able to relate the crack depth to the measurements of the horizontal and vertical displacements of the disc. They were taken during stationary operation at a rotational speed of $780 \mathrm{rev} / \mathrm{min}$. For the identification, the system was modeled according to eq. (22). But, of course, the dynamics of the test rig cannot be completely described by these equations, so that the covariance matrix of system noise $\mathrm{Q}$, eq. (12), has to be set to adequate values.

REKFIV and EKF estimate crack factors $r=0.012$ resp. $r=0.0178$ which correspond to a depth of the crack of $19.5 \%$ resp. $22.5 \%$, Mayes and Davies [1984]. The damage is overestimated, probably caused by the initiating slot, which prevents a complete closing of the crack surface. Figure 9 shows the identification of $r$ by REKFIV. The oscillations around the final value of $\mathrm{r}=$ 0.012 may be motivated by the fact, that the relatively long and thin shaft is somewhat prebent.

The calculations of the MEKF, figure 10, again provide us with a quantative result $(r \approx 0.013$; this corresponds to a crack depth of $20 \%$ ) and a qualitative result: the crack opens in the range $v_{1}(\tau)>0$ and the assumption that the crack surface really does not close completely is confirmed, because there is no range $v_{1}(\tau)$ $=0$ (compare to figure 5$)$.

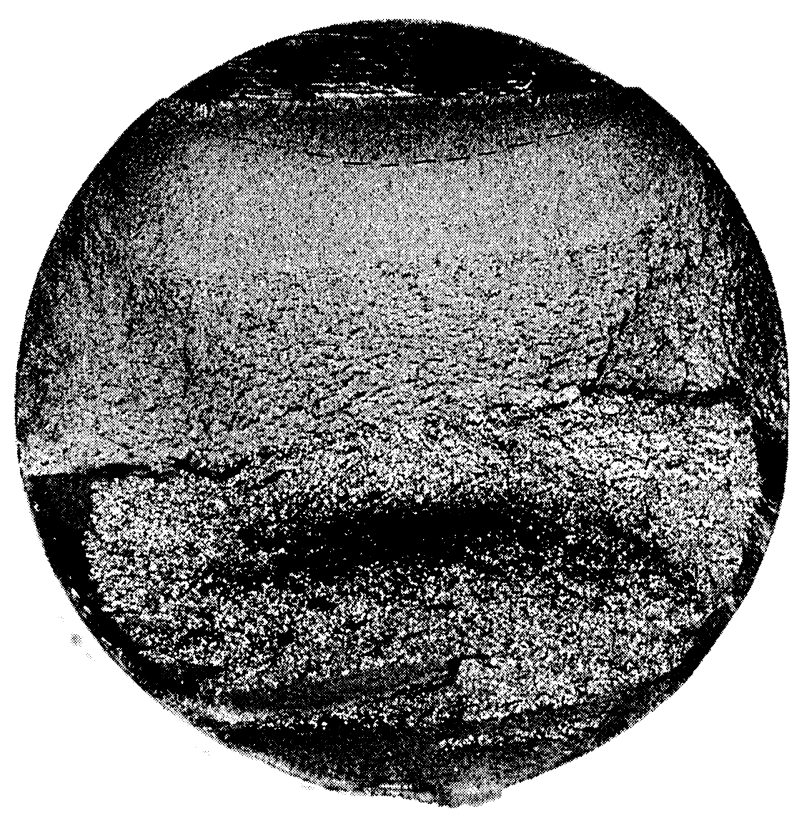

FIGURE 8 Fractured cross section and beach mark.

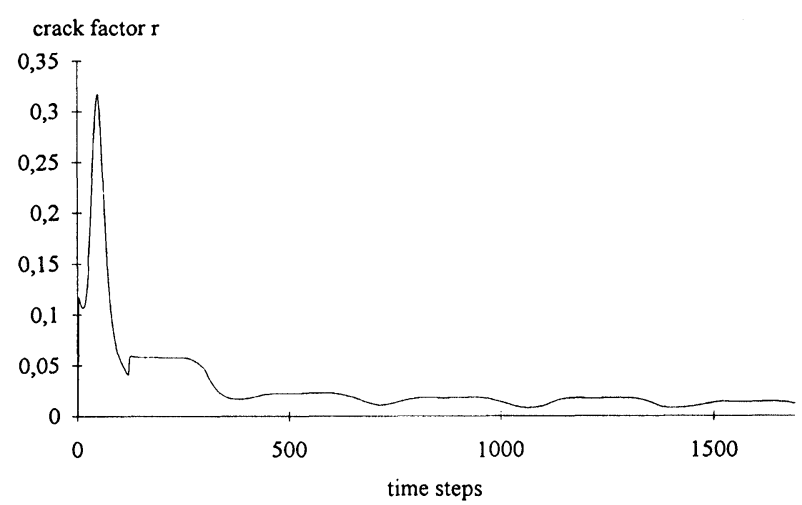

FIGURE 9 Identification of the crack factor $r$ by REKFIV; test rig.

\section{Rotor Test Rig: Identification of the Unbalance without Test Masses}

In the last example, EKF and REKFIV are utilized to determine the unbalance of the test rig described in the preceding chapter, with the modification that the diameter of the (undamaged) shaft is $12 \mathrm{~mm}$. Again, the horizontal and vertical vibrations of the disc are measured during stationary operation. The system was modeled according to eq. (22), setting $v(\tau)=0$. Table I shows the identified values of the unbalance parameters $\epsilon$ and $\beta$ and compares them to the results of a determination with test masses. The identification of the parameters by EKF is shown in figure 11.

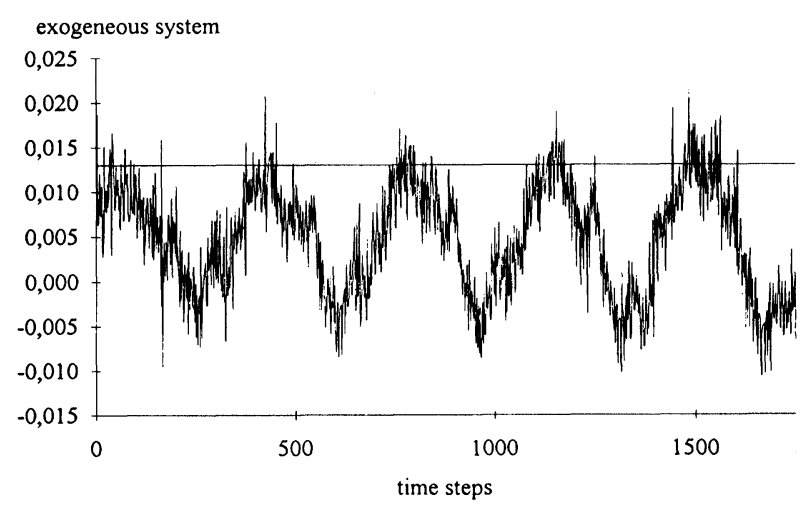

FIGURE 10 Identification of the nonlinear forces (exogeneous system) $v_{1}(\tau)$ due to the crack by MEKF; test rig.

TABLE I

Calculation of the unbalance of the test rig

\begin{tabular}{lll}
\hline & $\epsilon$ & $\beta$ \\
EKF & 0.073 & $0.11 \mathrm{rad}$ \\
REKFIV & 0.074 & $0.07 \mathrm{rad}$ \\
Calculation using test masses & 0.086 & $0 \mathrm{rad}$ \\
\hline
\end{tabular}




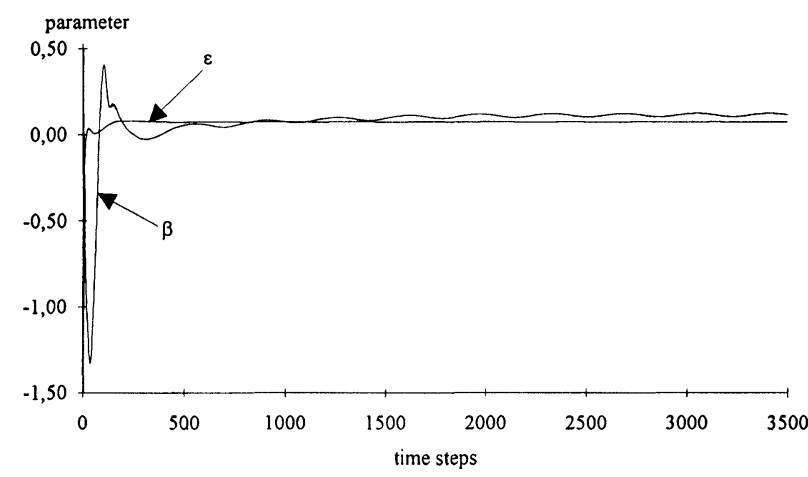

FIGURE 11 Identification of the unbalance parameters $\epsilon$ and $\beta$ by EKF; test rig.

\section{CONCLUSIONS}

System identification procedures offer the possibility to correct erroneous models, based on measurement data. Recently, this conventional field of application is being extended to fault detection and system diagnosis. In contrast to conventional approaches, identification procedures try to establish an unequivocal relation in between the damage and specific mechanical parameters, based on a suitable model. Furthermore, they can be employed during normal operation of the machinery.

In this paper, three system identification procedures were presented:

1. EKF: the Extended Kalman Filter as state and parameter estimator,

2. REKFIV: a recursive combination of the EKF as state estimator and the Instrumental Variables method,

3. MEKF: a modification of the EKF in analogy to the state observer designed by Müller [1990].

The feasability of the procedures for model-based fault detection of rotating machinery was proved by several examples. First, the crack depth of a simulated Jeffcott-rotor was calculated correctly. In addition, the results of the MEKF yielded a qualitative assertion about the character of the damage. Then, the three procedures were successfully applied to a rotor test rig, in order to detect the crack depth. A supplementary information was obtained: the crack surface did not close completely due to the initiating slot. Finally, it was shown that system identification procedures can be employed for the determination of unbalances without test masses.

In future projects, the algorithms will be extended in such a manner that they can be applied to larger structures modeled by finite elements.

\section{Acknowledgement}

The development of the combined algorithm REKFIV and the test rig is part of a project supported by the Deutsche Forschungsgemeinschaft (DFG), project no. Ha 1487/3-2.

\section{Nomenclature}

$\begin{array}{ll}E\{\ldots\} & \text { expected value of... } \\ x & \text { vector (true value) } \\ \hat{x} & \text { vector (estimated value) } \\ \dot{x} & \text { derivative of } x \text { with respect to } \mathrm{t} \\ x^{\prime} & \text { derivative of } x \text { with respect to } \tau \\ x^{\mathrm{T}} & \text { transpose of } x \\ A & \text { system matrix } \\ C & \text { measurement matrix } \\ I & \text { unity matrix } \\ K_{g} & \text { Kalman gain matrix } \\ p & \text { vector of parameters } \\ P & \text { covariance matrix } \\ Q & \text { covariance matrix of the system noise } \\ r & \text { crack factor } \\ R & \text { covariance matrix of the measurement noise } \\ t & \text { time } \\ u & \text { vector of inputs } \\ v & \text { vector of the external disturbances } \\ W & \text { Instrumental Variables matrix } \\ y & \text { measurement vector (outputs) } \\ z & \text { state space vector } \\ \epsilon & \text { error vector } \\ \epsilon, \beta & \text { related eccentricity and angle } \\ \tau & \text { dimensionless time } \\ & \end{array}$

\section{References}

Durbin, J., 1954. Errors in Variables, Revue de l'Institut International de Statistique, Vol. 22, pp. 23-32.

EL-Shafei, A., 1993. Measuring Vibrations for Machinery Monitoring and Diagnostics, Shock and Vibration Digest, Vol. 25, No. 1, pp. 3-14.

Eshleman, R.L., 1990. Detection, Diagnosis and Prognosis: an Evaluation of Current Technology, Proc. 44th Meeting of the Mechanical Failures Prevention Group, Virginia Beach, VA, pp. 33-42.

Fritzen, C.-P. and Seibold, S., 1990. Identification of Mechanical Systems by Means of the Extended Kalman Filter, Proc. 3rd International IFToMM Conf. on Rotordynamics, Lyon, France, pp. 423-429.

Gasch, R., 1976. Dynamic Behaviour of a Simple Rotor with a Cross-Sectional Crack, Conference on Vibrations in Rotating Machinery, IMechE Conference Publications, paper C178/76, University of Cambridge.

Imam, I., 1983. Method for on-line Detection of incipient Cracks in Turbine-Generator Rotors, US patent 4, 408, 294.

Jazwinski, A.H., 1970. Stochastic Processes and Filtering Theory, Academic Press, New York.

Jonas, O., 1992. Diagnostic Monitoring-An Overview, Power, 136, (1), pp. 61/108.

Kalman, R.E., 1960. A new Approach to Linear Filtering and Prediction Problems, Trans. ASME Series D, Journal of Basic Engineering, Vol. 82, pp. 35-45.

Ljung, L., 1979. Asymptotic Behaviour of the Extended Kalman Filter as a Parameter Estimator for Linear Systems, IEEE Trans. on Automatic Control, Vol. AC-24, No. 1, pp. 36-50. 
Mayes, I.W. and Davies, W.G.R., 1984. Analysis of the Response of a Multi-Rotor-Bearing System Containing a Transverse Crack in a Rotor, Journal of Vibration, Acoustics, Stress and Reliability in Design, paper 83-DET-84, pp. 139-145.

Müller, P.C., 1990. Indirect Measurements of Nonlinear Effects by State Observers, IUTAM-Symposium on Nonlinear Dynamics in Engineering Systems, Universität Stuttgart, Springer-Verlag, Berlin, pp. 205-215.

Muszynska, A., 1982. Shaft Crack Detection, Proc. 7th Machinery Dynamics Seminar, Edmonton, Canada, pp. 4.0-4.49.

Randall, R., 1990. Introduction to Condition Monitoring, Acoustics Australia, 18(1), pp. 15-18.

Seibold, S., Söffker, D. and Fritzen, C.-P., 1993. Modellgestützte Detektion von Wellenrissen, Dynamische Probleme-Modellierung und Wirklichkeit, Mitteilung des Curt-Risch-Institutes der Universität Hannover, Eds. H.G. Natke, H.K. Tönshoff, G. Meltzer, pp. 309-328

Seibold, S., Fritzen, C.-P. and Leifeld, A., 1993. A Combined State and Parameter Estimator Applied to Fault Detection, IUTAM-Symposium on Identification of Mechanical Systems, Universität Wuppertal, Springer Verlag, Berlin, Heidelberg, New York, to appear.

Söffker, D. and Bajkowski, J., 1991. Crack Detection of a Rotor by State Observers, Proc. of the 8th IFToMM World Congress on the Theory of Machines and Mechanisms, Prag, pp. 771-774.

Söffker, D., Yu, T. and Müller, P.C., 1994. State Estimation of Dynamical Systems with Nonlinearities by using Proportional-Integral Observer, Int. J. of Sys. Sc., to appear.

Waller, H. and Schmidt, R., 1990. The Application of State Observers in Structural Dynamics, Mechanical Systems and Signal Processing, 4(3), pp. 195-213.

Wauer, J., 1990. On the Dynamics of Cracked Rotors-a Literature Survey, Applied Mechanics Revue, Vol. 43, No. 1, pp. 13-17.

Wong, K.Y. and Polak, E., 1967. Identification of Linear Discrete Time Systems Using the Instrumental Variables Method, IEEE Trans. on Automatic Control, Vol. AC-12, No. 6, pp. 707-718.

Young, P., 1970. An Instrumental Variables Method for Real-Time Identification of a Noisy Process, Automatica, 6, pp. 271-287. 

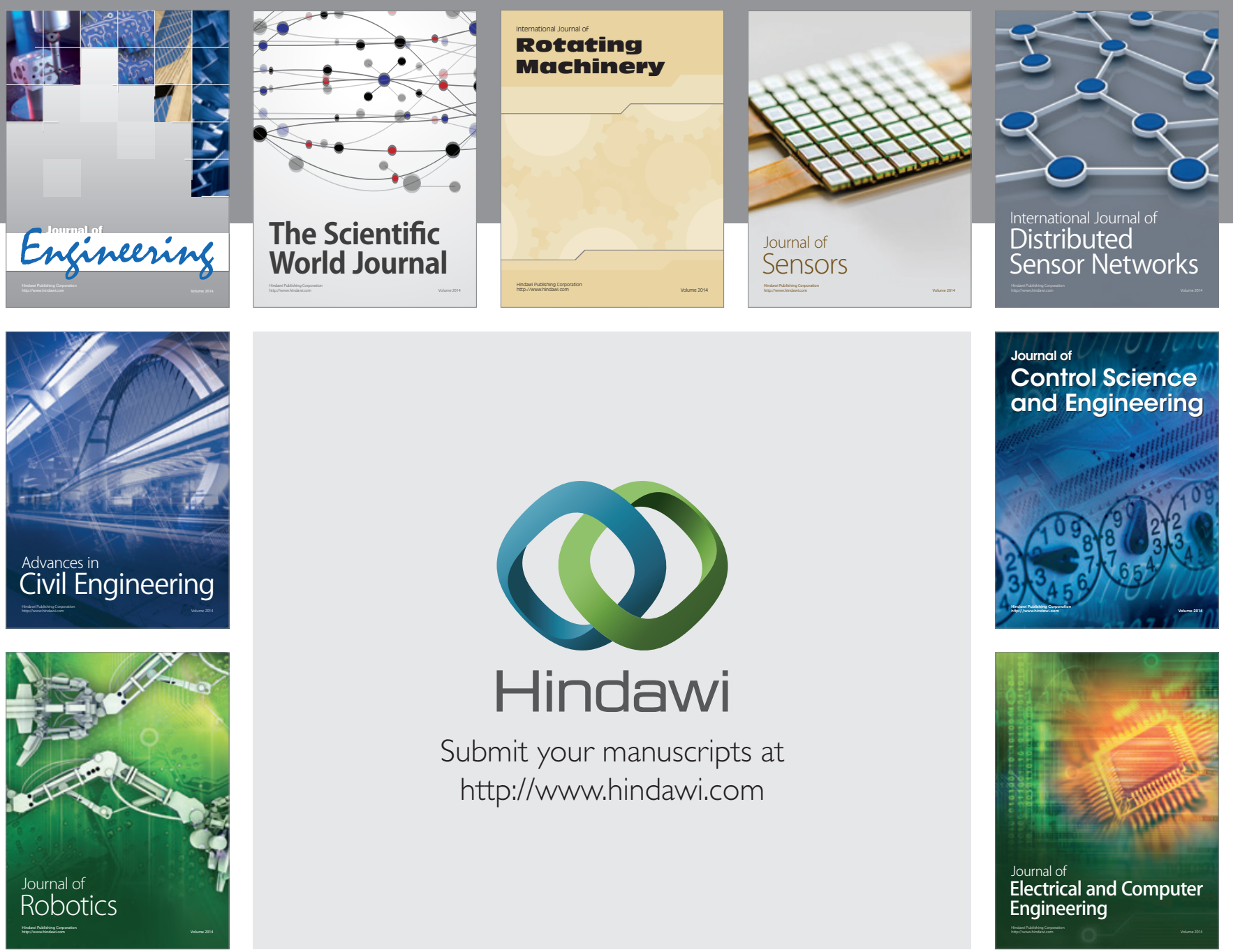

Submit your manuscripts at

http://www.hindawi.com
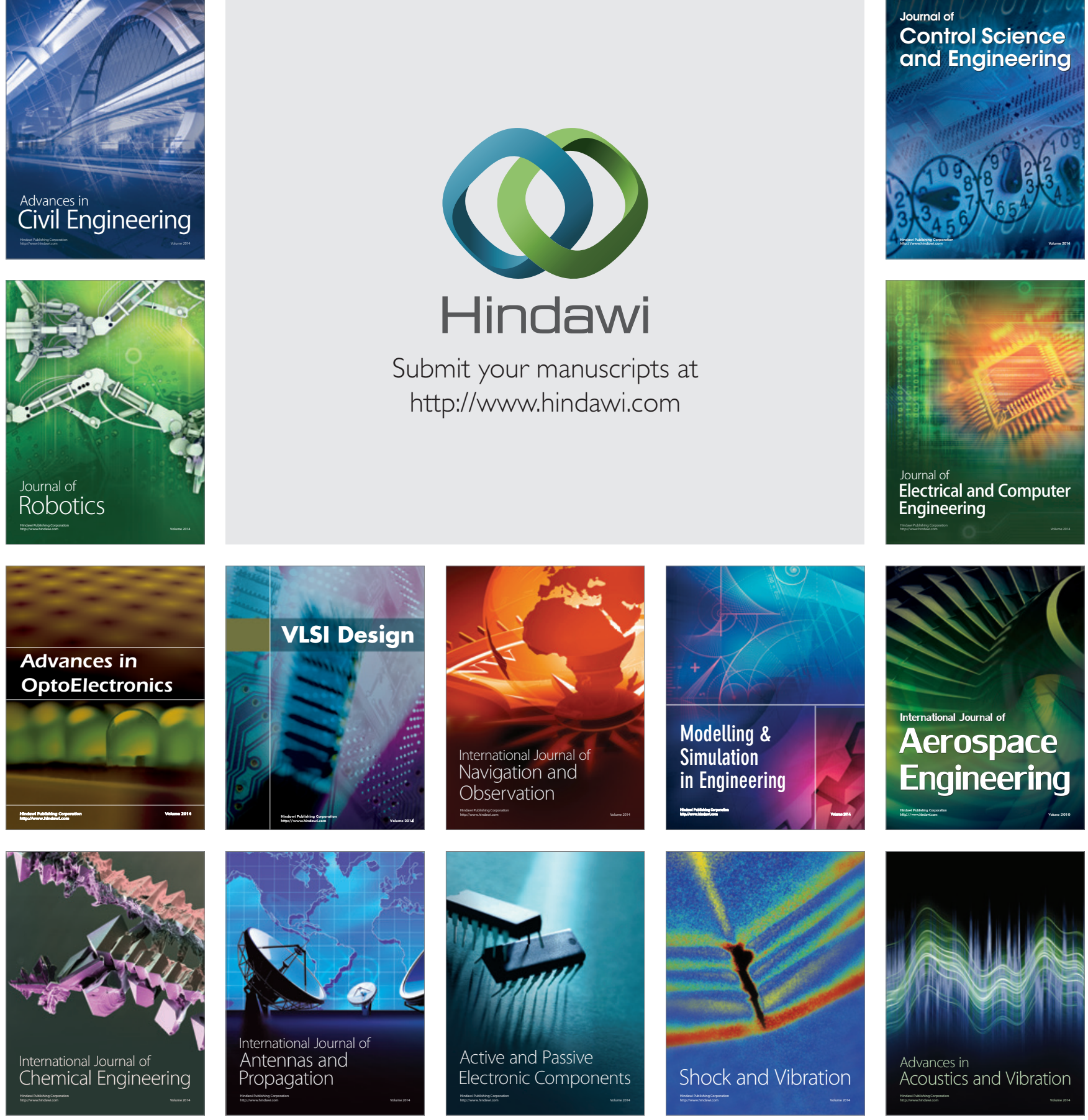